\title{
In Vivo Evaluation of Titanium Implants Bioactivated by a Modified Kokubo's Treatment
}

\author{
Jailson de Jesus, Enori Gemelli, Fábio Nery, Nelson H. A. Camargo \\ Materials Science and Engineering, Department of Mechanical Engineering, University of Santa Catarina State, \\ Campus Universitário, Joinville, Brazil \\ Email: nosliaj@gmail.com, enori@joinville.udesc.br, 2010fabionery@gmail.com, dem2nhac@joinville.udesc.br
}

Received December 2013

\begin{abstract}
In this study a modified Kokubo's treatment (MKT) was used to bioactivate titanium implants. Titanium surfaces were alumina-blasted and etched in $\mathrm{HCl} / \mathrm{H}_{2} \mathrm{SO}_{4}$ solution followed by treatment in autoclave at $120^{\circ} \mathrm{C}$ for $1,3,5$ hour periods with chemical solutions containing 1,2 and $3 \mathrm{M}$ of $\mathrm{NaOH}$. The materials were characterized by surface angle measurements, roughness, field emission scanning electron microscopy (FE-SEM), X-ray photoelectron spectroscopy (XPS), and in vivo tests. Statistical analysis by polynomial regression showed that solutions within 2.2 and $2.8 \mathrm{M} \mathrm{NaOH}$ lead to surface contact angles close to zero degree after $1 \mathrm{~h}$ of treatment. XPS analysis carried out on a sample treated $1 \mathrm{~h}$ in $2.2 \mathrm{M} \mathrm{NaOH}$ indicated the presence of a titanate film. This solution was selected to treat titanium implants for in vivo tests. These tests were performed on sheep in three types of separating surfaces: machined group (standard), SLA group (A) and MKT group (B) divided into two time intervals of 3 and 6 weeks. The samples in group $B$ displayed better results compared to group A only after 6 weeks in terms of counter torque and bone implant contact (BIC).
\end{abstract}

\section{Keywords}

Titanium; Thermochemical Treatment; Wettability; In Vivo Test

\section{Introduction}

It is well known that a series of interactions occur between the surface of biomaterials and the biological environment after they have been implanted into the human body [1]. The set of these interactions was called osseointegration, concept originally introduced by Brånemark et al. in 1977 [2]. The osseointegration is seen as a close contact between bone and implant [3], and the importance of the implant surface properties for a successful osseointegration was first pointed out by Albrektsson and co-workers [4]. The bone response, which means rate, quantity and quality are related to implant surface properties [5]. The composition and charges are critical for protein adsorption and cell attachment [6]. Hydrophilic surfaces seem to favor the interactions with biological fluids and cells when compared to the hydrophobic ones [7] [8], and hydrophilicity is affected by the surface 
chemical composition. Titanium and titanium alloys are frequently exploited as implant materials, because of their anticorrosive properties, mechanical properties, lightness and excellent biocompatibility of their surface oxides [9]. However, titanium is a bioinert material, i.e., the interface between titanium and host bone consists of simple interlocking attachment, without physic-chemical bonding with bone [10] [11]. Surface treatments are promising ways to improve titanium surface for dental implants in terms of chemical composition, energy level, morphology, topography and roughness [12]. Alkali treatment using $\mathrm{NaOH}$ solution is a well-recognized treatment to improve Ti implant surfaces for higher bone affinity. After treatment sodium titanate layer is produced on $\mathrm{Ti}$ surfaces where $\mathrm{TiO}_{2}$ hydrogel acts as nucleation regions for calcium phosphate precipitation [13]. During the alkali treatment, the passive oxide film is partially attacked by hydroxyl groups and dissolves into the alkali solution while hydration of Ti metal proceeds simultaneously. Negatively charged hydrates such as $\mathrm{HTiO}_{3}^{-}$. $\mathrm{nH}_{2} \mathrm{O}$ produced on the substrate surface combine with alkali ions from the aqueous solution to form an alkali titanate hydrogel layer [14]. A thermal treatment is usually carried out after alkali treatment. During the heating the hydrogel layer is dehydrated and densified to form a stable amorphous or crystalline titanate layer, this improves surface wettability of the implant [1]. Surface wettability influences implant osseointegration, since hydrophilic surfaces promote a conductive environment to bone formation, as evidenced by in vitro [15] and clinical tests [16]. After examining osteoblast response to controlled surface chemistries, Zhao and co-workers found that hydrophilic surfaces are osteogenic [8]. The Kokubo method is an effective treatment for bioactivation of titanium surface. However, the treatment produces a thick surface layer which may delaminate during implantation. Also, it is not an attractive treatment from practical viewpoint because it is a time consuming method involving chemical attack for a long period followed by a thermal treatment [17]. In this study a modified Kokubo treatment (MKT) was used to bioactivate titanium surfaces. This treatment is faster and exhibited capability to improve surface wettability and osseointegration of titanium implants.

\section{Material and Methods}

\subsection{Surface Treatment}

In this study solutions with varied amount of $\mathrm{NaOH}$ were used to treat titanium surfaces (Ti grade 4). The solutions were prepared with reagent grades containing $\mathrm{Ca}$, Na and $\mathrm{Si}$. The same base solution was used in the three situations, varying only the amount of $\mathrm{NaOH}$, which was 1,2 and $3 \mathrm{M} \mathrm{NaOH}$. The samples were treated in these solutions in an autoclave for 1 to 5 hours at $120^{\circ} \mathrm{C}$. Afterwards, the samples were rinsed with distilled water and dried in air. Titanium grade 4 discs of $6 \mathrm{~mm}$ diameter and $3 \mathrm{~mm}$ in length were blasted with alumina powder $(290 \mu \mathrm{m})$ and etched in $\mathrm{HCl} / \mathrm{H}_{2} \mathrm{SO}_{4}$ before the treatments. Three samples of each treatment were used for each surface characterization.

\subsection{Surface Characterization}

The sample surfaces were examined by contact angle measurements, FE-SEM (JSM-6701 F, JEOL, Tokyo, Japan), XPS and roughness. Using the sessile drop technique, contact angle measurements were taken of three samples of $6 \mathrm{~mm}$ in diameter of the as-treated materials. Liquid droplets of $2 \mu \mathrm{L}$ of deionized water were deposited onto the solid surfaces with a syringe. The contact angles were measured at room temperature $10 \mathrm{~s}$ after depositing the drop, which was the estimated time required to reach equilibrium with the liquid in use. A photo of the liquid droplet was taken with a digital camera and the contact angle was calculated by image analysis using ImageJ software. For statistical analyses of wettability a $3^{2}$ factorial design was used, varying the amount of $\mathrm{NaOH}(1,2,3 \mathrm{M})$ and the time of attack (1, 3 and $5 \mathrm{~h}$ ). The experimental design was designed by Statistica 8.0 (StatSoft, Inc., 2007) software. The XPS analyses were performed with a Phoibos 100 spectrometer (SPECS, Germany). The X-ray source was generated by MgKa (1253.6 eV), with $200 \mathrm{~W}$ power. The C1 s peak (284.6 eV) was used as an internal standard to correct the peak shifts caused by the accumulation of surface charge on insulating samples. The surface roughness was measured with a Mitutoyo SJ-201 profilometer.

\subsection{In Vivo Tests}

\subsubsection{Selected Implants}

Ninety six implants (diameter $=4.3 \mathrm{~mm}$, length $=13 \mathrm{~mm}$ ) of Ti grade 4 were included in this study. Sixty four samples were blasted with $\mathrm{Al}_{2} \mathrm{O}_{3}$ powder with an average particle size of $290 \mu \mathrm{m}$ and subsequently ultrasonical- 
ly cleaned in ethanol and water, dried in air, and etched with a solution of $\mathrm{HCl} / \mathrm{H}_{2} \mathrm{SO}_{4}$. The specimens were then divided into three equally sized groups $(\mathrm{n}=32)$. The first group was machined (surface standard-ST), the second group was SLA type (surface A) and the third group of blasted and acid etched implants from MKT (group B).

\subsubsection{Surgical Procedures}

The in vivo study comprised of 6 Finnish Dorset crossed-bred sheep (each weighing approximately 150 pounds) approximately 1.5 years old. The sheep remained in the facility for 2 weeks prior to the surgical procedures. A total of 96 implants were distributed among the experimental groups ( $\mathrm{n}=16$ per group and time-point). On each sheep, 8 implants were first placed in one side of the hip, and after 3 weeks the other side of the hip underwent the same procedure (16 samples per animal). Prior to the experimental procedures, the surgical area was shaved and aseptic procedures were done. The animals were then continuously monitored for heart rate, oxygen saturation, respiratory rate, temperature, and tissue coloration prior to complete shaving of the intended surgical site and the adjacent areas and application of a Poviodine solution. After monitoring, the animals were transferred over to an automated system, and aseptically draped. Anesthesia was inducted with Sodium Pentothal (15 - 20 $\mathrm{mg} / \mathrm{kg}$ ) in Normasol solution in the jugular vein. Anesthesia was maintained with Isoforane $(1.5 \%-3 \%)$ in $\mathrm{O}_{2} / \mathrm{N}_{2} \mathrm{O}$ (50/50). $500 \mathrm{mg}$ Cefazolin was administered pre-op intravenously and then again post-op. General anesthesia was maintained by the surgical staff of the vivarium. A circulating hot water blanket maintained body temperature while under anesthesia. ECG, End Tidal $\mathrm{CO}_{2}$ and $\mathrm{SpO}_{2}$ were monitored during the procedure. Implant placement was performed using drills prepared by the implant manufacturer.

\subsubsection{Removal Torque and Histologic Analysis}

The animals were euthanized after 6 weeks counting from the first implant surgery via anesthesia overdose, and the 3 and 6 weeks samples were retrieved by sharp dissection. Half of the implants were biomechanically tested (removal torque), while the remaining samples were referred to non-decalcified histology processing. In brief, the retrieved samples were dehydrated in a graded series of ethanol and thereafter were infiltrated and embedded in methylmethacrylate (MMA). The embedded resin blocks were then subjected to non-decalcified histologic sectioning where the samples were first perpendicularly sectioned into slices with a slow speed precision diamond saw (Isomet 2000, Buehler Ltd., Lake Bluff, USA). After gluing the sections to a plexi glass with a light curing acrylate-based adhesive (Technovit 7210 VLC adhesive, Heraeus Kulzer GMBH, Wehrheim, Germany), the samples were grinded and polished under abundant water irrigation using a series of silicon carbide (SiC) papers (400, 600, 800, 200) (Buehler Ltd., Lake Bluff, IL, USA) down to a final thickness of $30 \mu \mathrm{m}$. Thereafter, the sections were then stained in $1 \%$ toluidine blue. The bone-to-implant contact (BIC) along the implant was determined at $\times 50-200$ magnification (Leica DM2500M, Leica Microsystems GmbH, Wetzlar, Germany) by means of specialized computer software (Leica Application Suite, Leica Microsystems GmbH, Wetzlar, Germany). All evaluations concerning removal torque testing, histologic preparation, and histomorphometry was performed in a blinded manner.

\subsubsection{Statistical Analyses}

The effects of implant surface and time in vivo on removal torque and BIC were evaluated by a general linear ANOVA model (NCSS LLC). Statistical significance was set at 5\% $(\alpha=0.05)$.

\section{Results}

\subsection{Surface Characterization}

In accordance with statistical analyses of contact angle measurements displayed in Figure 1, treatments for $1 \mathrm{~h}$ with solutions containing 2.2 - 2.8 M NaOH lead to surface contact angles of approximately zero degree. Based on these results, surface characterization and in vivo tests were performed with samples treated with $2.2 \mathrm{M}$ $\mathrm{NaOH}$ for one hour (group B).

Figure 2 is a representative image of water drop behavior on surfaces of the different groups. The contact angles of group ST and A were found to be $63.10 \pm 1,25^{\circ}$ and $67.10 \pm 2,30^{\circ}$, respectively. As can be seen in Figure 2 the water drop was flat on the surface of group B, displaying a contact angle of zero degree. Figure 3 shows a sequence of water wettability on a Ti implant surface of group B. The water gradually rises as the surface becomes wet. 


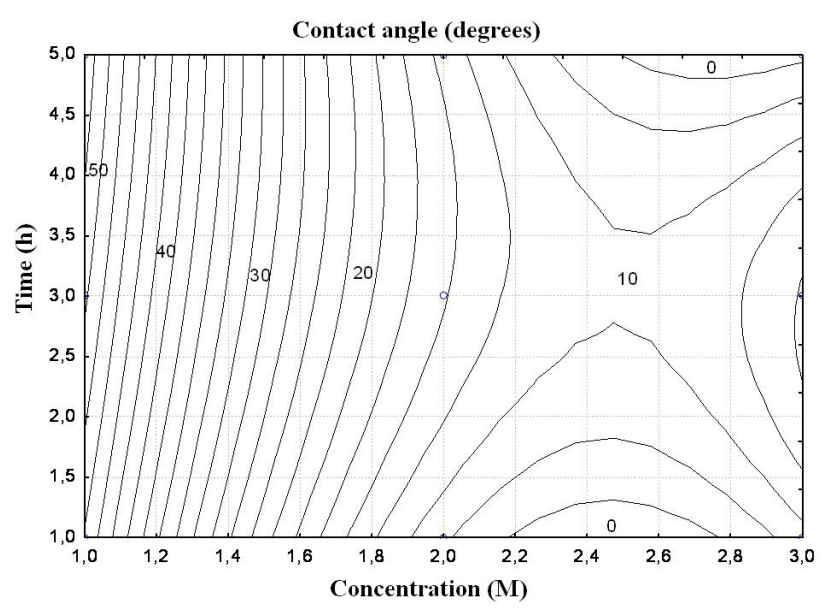

Figure 1. Contour plot of surface contact angle as a function of $\mathrm{NaOH}$ concentration and attack time.

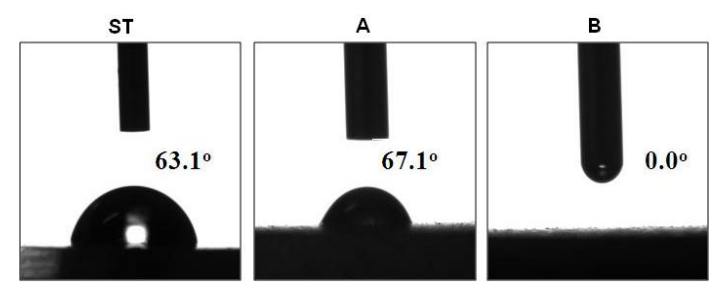

Figure 2. Surface contact angle of group ST, A and B.

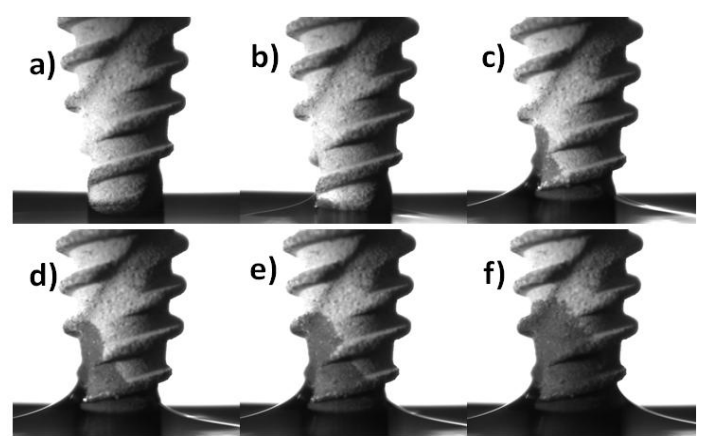

Figure 3. Wettability test of a Ti implant from group B.

FE-SEM observations revealed that machined samples (group standard) presented grooves coming from machining tools (Figure 4(a)). The group A samples (type SLA) presented macrorough surfaces structure formed after blasting and dual acid treatments (Figure 4(b)). After MKT treatment (group B), the implant surface showed finely irregular nanoporous structure (Figure 4(c) and (d)). According to XPS results this structure is composed of $\mathrm{Ti}, \mathrm{Na}, \mathrm{Ca}$ and $\mathrm{Si}$ (Figure 5).

As can be seen, the MKT treatment introduced elements which form bioactive phases/bonds. It is more likely that titanium surface is covered by a sodium and calcium titanate thin layer containing silicium. This change in morphology leads to a slight increase in roughness. The roughness of standard group was $0.20 \pm 0.04 \mu \mathrm{m}$, for group A was $1.93 \pm 0.23 \mu \mathrm{m}$ and for group B was $2.03 \pm 0.13 \mu \mathrm{m}$.

\subsection{Animal Model}

In the animal model, no signs of inflammation or infection were observed during the post-operatory phases and during sharp dissection following sacrifice. 


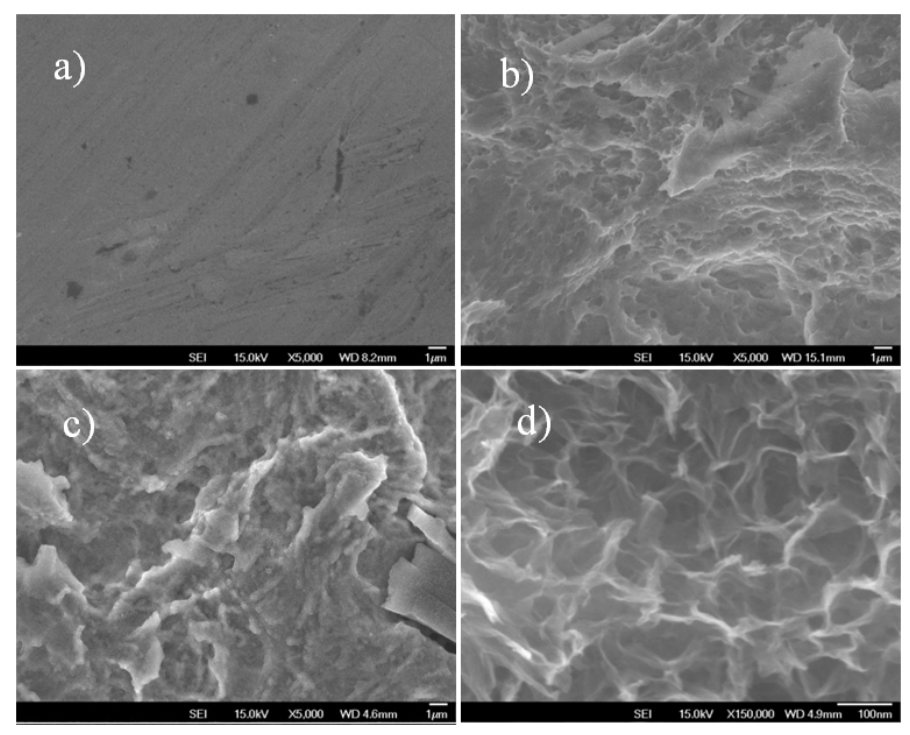

Figure 4. SEM images of different Ti surfaces (5 kx): (a) group St; (b) group A; (c) and (d-150 kx) group B.

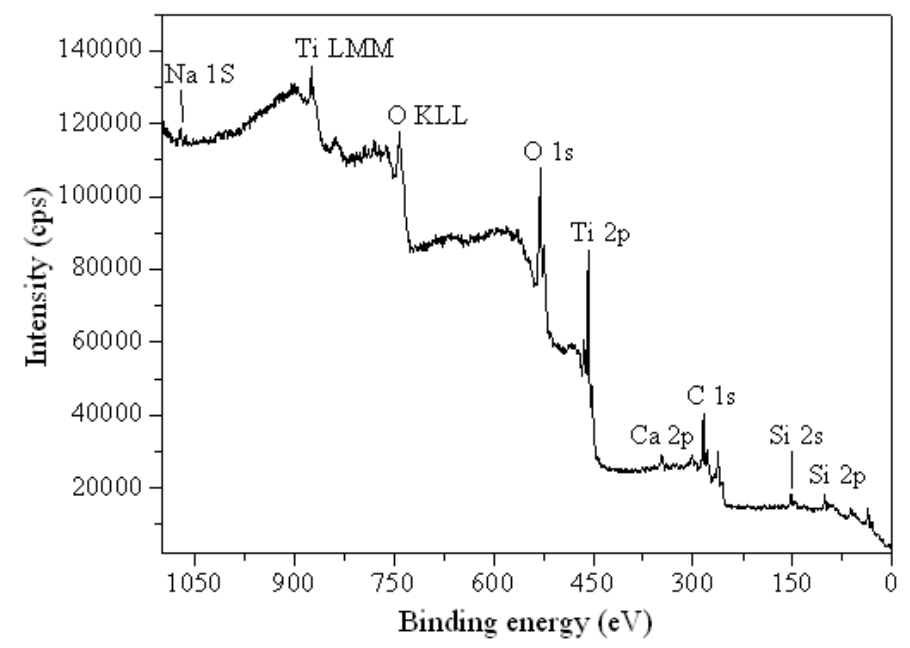

Figure 5. XPS binding energy of a sample from Group B.

\subsection{Removal Torque}

Both time in vivo and implant surface significantly affected removal torque values (both at $\mathrm{p}<0.001$ ). A significant increase in torque values were detected from 3 to 6 weeks in vivo when all surfaces were collapsed for analysis ( $\mathrm{p}<0.001)$. When surface treatment was evaluated by collapsing both times in vivo, significantly higher values were observed for the B surface group relative to the others $(p<0.001)$. A complete statistics summary presenting the estimated means and standard error for all surfaces at both times in vivo is presented in Figure 6. At first time in vivo A and B groups presented significantly higher removal torque values relative to standard group. At second time in vivo B group presented significantly higher removal torque relative to other groups. At 6 weeks, all groups presented significantly higher removal torque values relative to control.

\subsection{Histologic Observation}

Histologic observation presented bone formation in close contact to the implant surfaces for all groups at both time-points (Figure 7). However, the content of the bone seemed to differ depending on the surfaces, where higher degrees of interaction were observed for the experimental A and B surfaces relative to the standard 


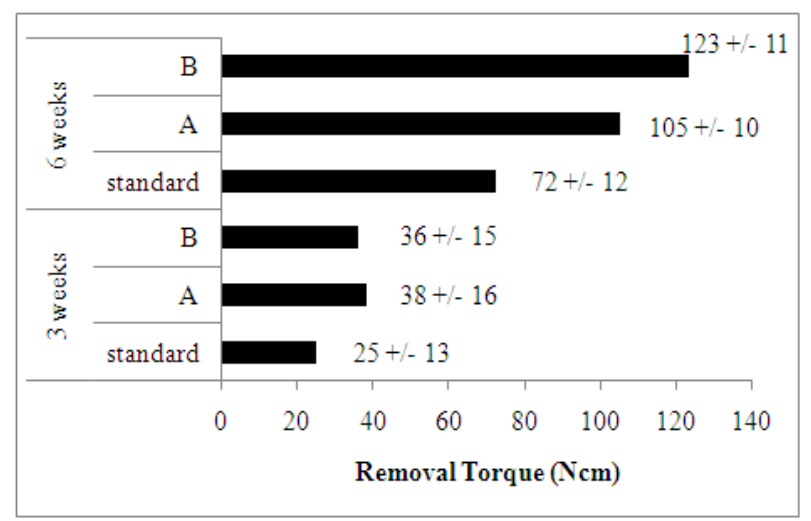

Figure 6. Statistics summary presenting the removal torque estimated means \pm standard error for the different surfaces at both times in vivo.

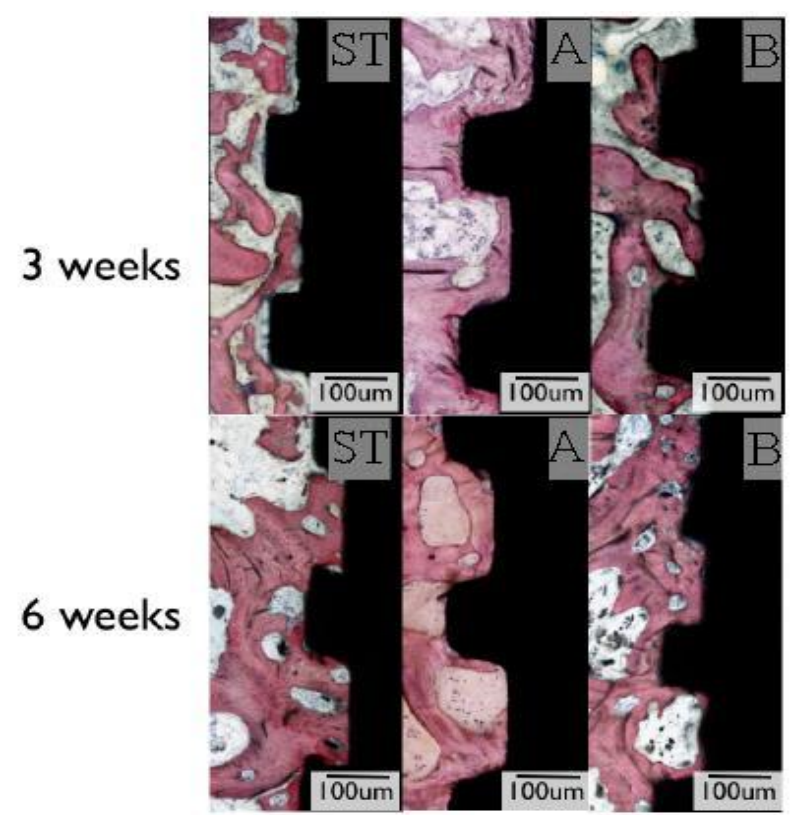

Figure 7. Representative histologic sections of all groups at 3 and 6 weeks in vivo.

as-machined control. At 6 weeks, the experimental B surface presented higher degrees of bone in contact to the implant. At 6 weeks, the bone in contact to the implant for all groups seemed to be higher compared to 3 weeks and the volume seemed to have increased compared to 3 weeks.

\subsection{Histomorphometric Evaluation-BIC}

The time in vivo significantly affected BIC values $(\mathrm{p}<0.001)$. The highest BIC values at 3 weeks were experimental A and B surface. At 6 weeks, the group B presented the highest value when compared to all other experimental groups. A significant increase in BIC values was detected from 3 to 6 weeks in vivo when all surfaces were collapsed for analysis $(\mathrm{p}<0.001)$. A complete statistics summary presenting the estimated means and standard error for all surfaces at both times in vivo is presented in Figure 8.

\section{Discussion}

The topography at the micrometer scale obtained by blasting and acid etching treatments increased osseointegration 


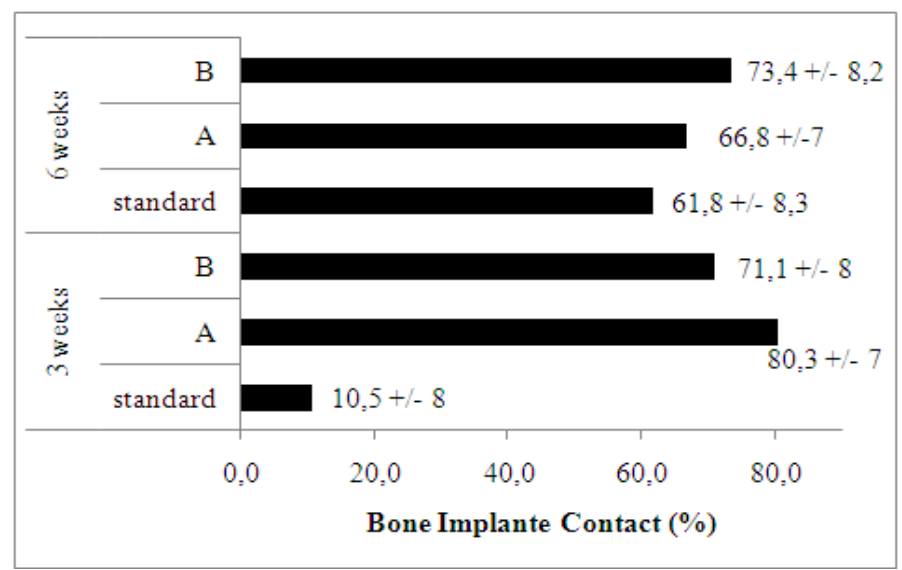

Figure 8. Statistics summary presenting the BIC estimated means \pm standard error for the different surfaces at both times in vivo.

by increasing surface roughness. The roughness is an important factor for cell adhesion. This process takes place by attachment of fibrins to the implant surfaces, which promote cell migration. After migration the implant surface becomes colonized by osteogenic cells prior to bone matrix production [18].

However, the SLA surfaces do not exhibit the capability to exchange ions with physiological medium. Ions exchanges are critical for a physic-chemical interaction of the implanted material with physiological medium. The mechanism of Ca-P deposition on alkali and heat treated titanium was proposed by Kokubo et al. [19]. When exposed to the medium, bioactive titanium generally releases the $\mathrm{Na}^{+}$ion from its sodium titanate surface into the medium via exchange with $\mathrm{H}_{3} \mathrm{O}^{+}$ions in the fluid, thereby forming many Ti-OH groups on its surface [19]. As a result, the surface is negatively charged, and then reacts with $\mathrm{Ca}^{+2}$ cations from the fluid to form a calcium titanate film. As the $\mathrm{Ca}^{+2}$ cations accumulate, the surface becomes positively charged, reacting with phosphate anions to form a bone-like apatite [19]. Chen et al. [20] investigated the release of $\mathrm{Na}^{+}$ions from $\mathrm{NaOH}$-treated titanium with and without a $1 \mathrm{~h}$ heat treatment at $600^{\circ} \mathrm{C}$. The specimens were immersed in a buffer solution at biological temperature and $\mathrm{pH}$ and the release of $\mathrm{Na}^{+}$ions into the buffer solution was measured after 1, 3, 5 and 7 days. They found that the $\mathrm{Na}^{+}$concentration increased sharply in the buffer solution, and that the amount of this increase in $\mathrm{NaOH}$ treated titanium was higher than in $\mathrm{NaOH}$ heat-treated titanium. The $\mathrm{Na}^{+}$ ion concentration reached its highest dissolution level of 0.20 and $0.55 \mathrm{mM}$ after 1 - 3 days in samples with and without heat treatment, respectively. The sodium titanate appeared to become more stable after heating, hindering the release of $\mathrm{Na}^{+}$ions. In this study the titanate film produced by MKT treatment is composed of sodium and calcium. The presence of calcium in the film may be an advantage compared to the Kokubo treatment because it may accelerate apatite formation, since apatite formation depends on the formation of a calcium titanate layer. The titanate layer produced by the MKT treatment is less stable than that formed by $\mathrm{NaOH}$ heat-treated at $600^{\circ} \mathrm{C}$. Thus, a higher ions exchange is expected for MKT treatment, which may accelerate apatite formation on titanium implants. The in vivo results showed that the MKT treatment (group B) accelerated osseointegration compared to the other groups. The hydrophilicity of group B is attributed to the formation of Ti-OH bonds on the surface.

\section{Conclusion}

The MKT treatment was demonstrated to be efficient for bioactivation of titanium implants. A completely hydrophylic surface was achieved by $1 \mathrm{~h}$ treatment in autoclave at $120^{\circ} \mathrm{C}$ with a solution containing $2.2 \mathrm{M} \mathrm{NaOH}$. The treatment produced a thin titanate layer composed of sodium, calcium and silicium. This microstructure promoted physic-chemical interactions between biological medium and titanium surface. These interactions increased cell proliferation and osseointegration as evidenced by the in vivo tests.

\section{References}

[1] Liu, X., Chu, P.K. and Ding, C. (2010) Surface Nano-Functionalization of Biomaterials. Materials Science and Engi- 
neering R, 70, 275-302. http://dx.doi.org/10.1016/j.mser.2010.06.013

[2] Brånemark, P.I., Hansson, B.O., Adell, R., Breine, U., Lindström, U., Hallén, O. and Oman, A. (1977) Osseointegrated Implants in the Treatment of the Edentulous Jaw. Experience from a 10-Year Period. Scandinavian Journal of Plastic and Reconstructive Surgery, 16, 7-127.

[3] Albrektsson, T. and Wennerberg, A. (2004) Oral Implant Surfaces: Part 1-Review Focusing on Topographic and Chemical Properties of Different Surfaces and in Vivo Responses to Them. International Journal of Prosthodontics, 17, 536-543.

[4] Albrektsson, T., Brånemark, P.I., Hansson, H.A. and Lindström, J. (1981) Osseointegrated Titanium Implants. Acta Orthopaedica Scandinavica, 52, 155-179. http://dx.doi.org/10.3109/17453678108991776

[5] Novaes Jr., A.B., Souza, S.L.S., Barros, R.R.M., Pereira, K.K.Y., Iezzi, G. and PiattellI, A. (2010) Influence of Implant Surfaces on Osseointegration. Brazilian Dental Journal, 21, 471-481.

[6] Junker, R., Dimakis, A., Thoneick, M. and Jansen, J.A. (2009) Effects of Implant Surface Coatings and Composition on Bone Integration: A Systematic Review. Clinical Oral Implants Research, 20,185-206. http://dx.doi.org/10.1111/j.1600-0501.2009.01777.x

[7] Buser, D., Broggini, N., Wieland, M., Schenk, R.K., Denzer, A.J., Cochran, D.L., Hoffmann, B., Lussi, A. and Steinemann, S.G. (2004) Enhanced Bone Apposition to a Chemically Modified SLA Titanium Surface. Journal of Dental Research, 83, 529-533. http://dx.doi.org/10.1177/154405910408300704

[8] Zhao, G., Schwartz, Z., Wieland, M., Rupp, F., Geis-Gerstorfer, J., Cochran, D.L. and Boyan, B.D. (2005) High Surface Energy Enhances Cell Response to Titanium Substrate Microstructure. Journal of Biomedical Materials Research Part A, 74, 49-58. http://dx.doi.org/10.1002/jbm.a.30320

[9] Rihova, Z., Stary, V. and Bacakova, L. (2012) A Study of the Structure and Surface Properties of Nanostructured Biocompatible Coatings on Ti Alloys. Vacuum, 86, 630-633.

[10] Lu, X., Zhao, Z. and Leng, Y. (2007) Biomimetic Calcium Phosphate Coatings on Nitric-Acid-Treated Titanium Surfaces. Materials Science and Engineering C, 27, 700-708. http://dx.doi.org/10.1016/j.msec.2006.06.030

[11] Kilpadi, D.V. and Lemons, J. E. (1994) Surface Energy Characterization of Unalloyed Titanium Implants. Journal of Biomedical Materials Research, 28, 1419-1425. http://dx.doi.org/10.1002/jbm.820281206

[12] Rupp, F., Scheideler, L., Olshanska, N., de Wld, M., Wieland, M. and Geis-Gesttorfer, J. (2006) Enhancing Surface Free Energy and Hydrophilicity through Modification of Microstructured Titanium Implant Surface. Journal of Biomedical Materials Research, 76, 323. http://dx.doi.org/10.1002/jbm.a.30518

[13] An, S.-H., Matsumoto, T., Miyajima, H., Sasaki, J.-I., Narayanan, R. and Kim, K.-H. (2012) Surface Characterization of Alkali- and Heat-Treated Ti with or without Prior Acid Etching. Applied Surface Science, 258, 4377-4382. http://dx.doi.org/10.1016/j.apsusc.2011.12.119

[14] Kim, H. M., Miyaji, F., Kokubo, T. and Nakamura, T. (1996) Preparation of Bioactive Ti and Its Alloys via Simple Chemical Surface Treatment. Journal of Biomedical Materials Research, 32, 409-417. http://dx.doi.org/10.1002/(SICI)1097-4636(199611)32:3<409::AID-JBM14>3.0.CO;2-B

[15] Park, J.H., Wasilewski, C.E., Almodovar, N., Olivares-Navarrete, R., Boyan, B.D., Tannenbaum, R. and Schwartz, Z. (2012) The Responses to Surface Wettability Gradients Induced by Chitosan Nanofilms on Microtextured Titanium Mediated by Specific Integrin Receptors. Biomaterials, 33, 7386-7393. http://dx.doi.org/10.1016/j.biomaterials.2012.06.066

[16] Bornstein, M.M., Wittneben, J.G., Bragger, U. and Buser, D. (2010) Early Loading at 21 Days of Non-Submerged Titanium Implants with a Chemically Modified Sandblasted and Acid-Etched Surface. 3-Year Results of a Prospective Study in the Posterior Mandible. Journal of Periodontology, 81, 809-818. http://dx.doi.org/10.1902/jop.2010.090727

[17] Kokubo, T. and Yamaguchi, S. (2010) Novel Bioactive Titanate Layers Formed on Ti Metal and Its Alloys by Chemical Treatments. Materials, 3, 48-63. http://dx.doi.org/10.3390/ma3010048

[18] Davies, J.E. (2003) Understanding Peri-Implant Endosseous Healing. Journal of Dental Education, 67, $932-949$.

[19] Kokubo, T., Matsushita, T. and Takadama, H. (2007) Titania-Based Bioactive Materials. Journal of the European Ceramic Society, 27, 1553-1558. http://dx.doi.org/10.1016/j.jeurceramsoc.2006.04.015

[20] Chen, Y., Zheng, X., Ji, H. and Ding, C. (2007) Effect of Ti-OH Formation on Bioactivity of Vacuum Plasma-Sprayed Titanium Coating after Chemical Treatment. Surface and Coatings Technology, 202, 494-498.

http://dx.doi.org/10.1016/j.surfcoat.2007.06.015 